\title{
Síndrome de Down: uma revisão integrativa da literatura (2005-2020)
}

\author{
Down Syndrome: an integrative literature review (2005-2020) \\ Síndrome de Down: una revisión de la literatura integradora (2005-2020)
}

\author{
Rafael Soares Silva \\ ORCID: https://orcid.org/0000-0001-9994-6653 \\ Universidade de São Paulo, Brasil \\ E-mail: silva.rafael@usp.br \\ Thayane Nascimento Freitas \\ ORCID: https://orcid.org/0000-0002-0456-5039 \\ Secretaria Municipal de Educação, Brasil \\ E-mail: thayanny_freitas@hotmail.com \\ Nádia Fernanda Martins de Araújo \\ ORCID: https://orcid.org/0000-0001-6627-4862 \\ Universidade Federal do Piauí, Brasil \\ E-mail: nadiaaraujo1@hotmail.com \\ Tarcísio Welvis Gomes de Araújo \\ ORCID: https://orcid.org/0000-0002-6157-1769 \\ Secretaria Municipal de Educação, Brasil \\ E-mail:twgaraujo@gmail.com \\ Izabel Rodrigues da Silva \\ ORCID: https://orcid.org/0000-0003-3094-3379 \\ ATSD-PI, Brasil \\ E-mail: izabel6418@gmail.com
}

\begin{abstract}
Resumo
A pessoa com Síndrome de Down (SD) apresenta uma alteração no número de autossomos, apresentando particularidades físicas, motoras, cognitivas e linguísticas, sendo necessárias intervenções específicas para que as habilidades do sujeito sejam desenvolvidas de forma significativa. Deste modo, os objetivos deste artigo consistem em revisar a literatura que discutem a despeito das dificuldades que passam ou que possam interferir na aquisição e desenvolvimento da linguagem da pessoa com Síndrome de Down. Na metodologia foi realizada uma Revisão Integrativa (RI) de literatura sobre aspectos, tendências e avanços relacionados à Síndrome de Down publicados na Revista Brasileira de Educação Especial no período que consiste entre 2005 à 2020. Pôde-se constatar que as crianças com SD apresentam consideráveis dificuldades no desenvolvimento da linguagem, bem como da família e dos professores em lidar com esse sujeito, assim, é indispensável que a família, escola e outros profissionais que acompanham a criança com Down construam uma relação baseada no trabalho colaborativo, de modo que os diversos saberes sejam considerados possibilitando situações de aprendizagem significativa no que cerne a aquisição da linguagem. Dentre as estratégias identificadas ressaltamos: a importância da família na construção de diálogos; influência da leitura diária para que a criança aumente seu vocabulário e compreenda as organizações sintáticas da língua; trabalho com gêneros textuais pelos professores; possibilitar à criança com SD o contato com outras crianças com e sem a síndrome, para que tenham contato com diferentes linguagens por meio de brincadeiras, jogos, danças e músicas; caso necessário, fazer uso de comunicação alternativa.
\end{abstract}

Palavras-chave: Síndrome de Down; Linguagem; Literatura de Revisão.

\begin{abstract}
The person with Down Syndrome (DS) presents an alteration in the number of autosomes, physical, motor, cognitive and linguistic particularities, requiring a specific requirement for the subject's abilities to be developed in a reinforced way. Thus, the objectives of this article are to review the literature that they discuss despite the difficulties that they experience or that may interfere with the acquisition and development of language in a person with Down Syndrome. In the methodology, an integrative literature review (IR) was carried out on aspects, trends and advances related to Down Syndrome published in the Brazilian Journal of Special Education in the period between 2005 and 2020. It was found that children with DS present considerable difficulties in the development of language, as well as of the family and teachers in dealing with this subject, thus, it is essential that the family, school and other professionals who accompany the child with Down build a relationship based on collaborative work, so that the various knowledge are considered enabling learning hypotheses regarding language acquisition. Among the identified identified, we highlight: an importance of the family in the construction of dialogues; inclusion of daily reading for the child to increase their vocabulary and understand the syntactic associations of the language; work with textual genres by teachers; enable
\end{abstract}


children with DS to have contact with other children with and without the syndrome, so that they can have contact with different languages through games, games, dances and music; if necessary, make use of alternative communication.

Keywords: Down's Syndrome; Language; Review Literature.

\begin{abstract}
Resumen
La persona con Síndrome de Down (SD) presenta una alteración en el número de particularidades autosomas, físicas, motoras, cognitivas y lingüísticas, requiriendo un requerimiento específico para que las habilidades del sujeto se desarrollen de manera reforzada. Así, los objetivos de este artículo son revisar la literatura que discuten a pesar de las dificultades que experimentan o que pueden interferir con la adquisición y desarrollo del lenguaje en una persona con Síndrome de Down. En la metodología, se realizó una revisión integradora de la literatura (RI) sobre aspectos, tendencias y avances relacionados con el Síndrome de Down publicados en la Revista Brasileña de Educación Especial en el período 2005-2020. Se encontró que los niños con SD presentan dificultades considerables en el desarrollo del lenguaje, así como de la familia y docentes en el abordaje de este tema, por ello, es fundamental que la familia, la escuela y demás profesionales que acompañan al niño con Down construyan una relación basada en el trabajo colaborativo, de manera que él se consideran diversos conocimientos como hipótesis de aprendizaje habilitantes en relación con la adquisición del lenguaje. Entre los identificados, destacamos: la importancia de la familia en la construcción de diálogos; inclusión de la lectura diaria para que el niño aumente su vocabulario y comprenda las asociaciones sintácticas del idioma; trabajar con géneros textuales por parte de los profesores; posibilitar que los niños con SD tengan contacto con otros niños con y sin síndrome, para que puedan tener contacto con diferentes idiomas a través de juegos, juegos, bailes y música; si es necesario, utilice la comunicación alternativa.
\end{abstract}

Palabras clave: Síndrome de Down; Idioma; Revisión de la literatura.

\title{
1. Introdução
}

Atualmente a Educação Especial na perspectiva Inclusiva é um dos temas mais discutidos no campo educacional e sua importância levou a promulgação da Lei Brasileira de Inclusão (também conhecida como Estatuto da Pessoa com Deficiência) e a sua inserção nas propostas de políticas públicas, nas leis civis e educacionais do Brasil como a Constituição Federal, a Lei de Diretrizes e Bases da Educação Nacional (LDB 9394/96), o Estatuto da Criança e do Adolescente (ECA 8.069/90) e as várias políticas de atendimento à pessoa com deficiência propostas pelo governo federal (Silva \& Amaral, 2020).

Através das pesquisas científicas podemos abstrair conhecimentos que mudam a vida de qualquer indivíduo, seu modo de ser, agir e se comportar no meio social. Pode-se ainda reduzir preconceitos, criar estratégias objetivando superar déficits cognitivos, medos e ansiedades e promover um processo de ensino-aprendizagem adequado visando aprendizagens significativas do estudante conforme suas singularidades.

Toda sociedade humana tem uma linguagem e é impossível imaginar que alguma funcione sem a linguagem, visto que esta é transmitida de uma geração a outra através do meio escrito ou oral. Logo, são transmitidos conhecimentos, cultura e crenças, pois a linguagem afeta todos os aspectos do comportamento humano e a construção da identidade do indivíduo.

Assim, essa pesquisa se justifica pela necessidade em compreender quem é a pessoa com Síndrome de Down (SD), suas características linguísticas, cognitivas, sociais, físicas de modo a possibilitá-las processos de desenvolvimentos condizentes com suas habilidades linguísticas.

Diante do exposto, este estudo teve como objetivo revisar a literatura a despeito das dificuldades que passam ou que possam interferir na aquisição e desenvolvimento da linguagem da pessoa com Síndrome de Down, visto que a linguagem é de um todo essencial nas nossas vidas e saber lidar com essas interferências cognitivas, impacta a forma sobre a qual olhamos e lidamos os modos de ser, agir e se comportar desses sujeitos.

Para se alcançar o objetivo proposto a metodologia utilizada consistiu em uma Revisão Integrativa (RI) de literatura sobre aspectos, tendências e avanços relacionados à Síndrome de Down publicados na Revista Brasileira de Educação Especial no período que consiste entre 2005 à 2020.

\subsection{Particularidades da Trissomia 21}

A síndrome de Down é causada pela alteração no número normal de autossomos, ou seja, presença de três cromossomos 
no par 21 (trissomia do par 21) em todas ou na maior parte das células de um indivíduo. Essa aberração autossômica acontece no momento da concepção do feto, ou seja, ocorre a trissomia do cromossomo 21, em que a pessoa possui 47 cromossomos em suas células em vez de 46, como a maior parte da população.

A divisão celular nas crianças com Síndrome de Down revela uma distribuição defeituosa dos cromossomas. Assim, a criança "normal" recebe 23 pares específicos de cromossomas (Martinho, 2011). Sabe-se que a incidência de desordens cromossômicas aumenta com a idade avançada da mãe, aproximadamente, após os 35 anos de idade.

A condição está geralmente associada a atraso no desenvolvimento infantil, feições faciais características (forma de lua cheia), inchaço das pálpebras, falta de coordenação motora e quociente intelectual leve a moderado.

Dessa maneira, as características físicas de crianças com Síndrome de Down são formadas por influências de seu material genético e alguma destas podem se apresentar de forma mais acentuada em algumas crianças do que em outras, mas isso não quer dizer que todas as crianças são iguais, pois as características vão se modificando com o passar do tempo (Pueschel, 1993).

Com isso, observa-se o atraso em seu desenvolvimento cognitivo (característica mais constante da SD) com alterações significativas na atenção e memória de curto prazo, o que dificulta a conservação de informações imediatas, o que leva ao comprometimento tanto da memória visual como auditiva, pois constituem prejuízos nas regiões corticais e subcorticais, incluindo o cerebelo - áreas cruciais para o desenvolvimento da linguagem. Logo, são questões que precisam ser corrigidas de forma precoce e ao longo do desenvolvimento, pois o mau funcionamento dessas regiões reflete de forma negativa sobre o processo de entrada de informação e o seu posterior processamento cerebral.

\subsection{A linguagem na Síndrome de Down}

A linguagem é a forma que utilizamos para comunicar, representar e transmitir nossos pensamentos, anseios e sentimentos, através de signos convencionais, sonoros, gráficos e gestuais. Logo, a linguagem é parte fundamental em nossas vidas e nos acompanha desde que nascemos, e prolonga-se durante toda a vida do indivíduo.

Representa assim, um dos aspectos mais importantes a ser desenvolvido por qualquer criança, para que possa se relacionar com as demais pessoas e integrar-se no seu meio social, pois o "dizer" estar em todas as nossas ações diárias, desde uma roupa que vestimos até o nosso choro.

Na criança com Síndrome de Down a linguagem se estrutura nas relações com os outros, isto é, a criança precisa de um orientador/interlocutor que através do seu discurso colaborará bastante para que a linguagem se estruture e seja adquirida de forma eficaz. Desse modo, sabendo que cada pessoa, família ou sociedade tem suas peculiaridades, a criança trissômica também apresenta suas especificidades, apesar de apresentarem muitas características parecidas e, não podemos dizer encontrar um indivíduo igual ao outro se analisarmos detalhadamente a linguagem destes (Schwartzman, 2003).

Nesse ínterim, alguns fatores podem interferir de forma diferenciada na aquisição da linguagem, como os cognitivos, emocionais e físicos, mas apenas influenciam e não determinam. Assim, essas crianças escolherão o melhor meio de comunicação para si, em um determinado período de seu desenvolvimento, ou seja, a palavra tem poder para exteriorizar os desejos, os pensamentos e as emoções de forma que para o adulto/orientador é decodificada mais facilmente, mas todas as formas de dizer constituem-se como instrumento da linguagem, o que estrutura a condição de sujeito.

Sobre isso, esclarece-nos Schwartzman (2003, p. 23) que:

O processo de aprendizagem é contínuo, tendo início já a partir do nascimento com a experiencia do sugar, tocar, virarse e erguer a cabeça, em conjunto com o olhar e o ouvir. Entretanto, com a ajuda adequada, a aprendizagem ocorre em ritmo mais lento. 
Desta forma, a criança com Síndrome de Down apresenta dificuldade na aquisição e desenvolvimento da linguagem como processo complexo, se desenvolvendo de modo mais lento e mostrando uma certa complicação para se comunicar com clareza, se comparada com desenvolvimento de outra criança sem SD. Apresentam, assim, atrasos consideráveis de linguagem e necessitam de intervenção para corrigir.

De certo, tem-se a linguagem como um dos aspectos mais importantes a ser desenvolvido por qualquer criança, para que possa relacionar-se com as demais pessoas e integrar-se ao meio social, adquirindo os conhecimentos historicamente construídos.

Sendo assim, as pessoas com maiores habilidades na linguagem podem transmitir de forma mais fácil os seus sentimentos, desejos e pensamentos, pois a aquisição e desenvolvimento da linguagem implica: aprender a combinar fonemas, palavras e frases em sequências compreensíveis para os outros; conhecer e compartilhar os significados elaborados sócioculturalmente por uma determinada comunidade linguística e saber utilizar corretamente as regras gramaticais que estruturam as relações forma-função na linguagem.

Consoante, Piaget (1999) pondera que as estruturas da linguagem não estão predispostas desde o nascimento, nem são dadas pelo meio ambiente, isto é, essas estruturas são construídas por cada criança enquanto interagem com o meio, nas relações com sociais, com outros sujeitos, pois vão adquirindo e selecionando conhecimentos que lhes vão permitir construir estruturas conceituais que mais tarde levam ao desenvolvimento de uma estrutura linguística.

Por outro lado, Vygotsky (1997) destaca que o princípio geral que rege o desenvolvimento "normal" não está em desacordo para aquele que apresenta deficiência intelectual. Segundo ele, a criança com deficiência se desenvolve de outras maneiras, ou seja, o ser humano tem a capacidade de se adaptar às mais diversas situações a que está exposto, ainda que exposto a condições adversas.

Logo, Vygotsky (1997, p. 14 como citado em Filho, 2012, p. 8) menciona que o desenvolvimento é o mesmo para as crianças com ou sem deficiência, mas que as limitações mediadas pela deficiência funcionam como um elemento motivador. A saber:

Todo defeito cria estímulos para elaboração de uma compensação. Por isso, o estudo da criança deficiente não pode se limitar a determinar o nível e a gravidade da insuficiência e, sim, incluir obrigatoriamente a consideração dos processos compensatórios, quer dizer, substitutivos, superestruturados e niveladores, no desenvolvimento e na conduta da criança.

Percebe-se que a utilização de recursos técnicos e metodologias específicas permitem ao sujeito superar suas limitações e criar outras vias que garantam a sua inclusão na vida produtiva em plena colaboração com os não deficientes. Por isso, a sociedade pode diminuir e/ou eliminar os limites que a deficiência tem imposto, especialmente pela via de uma Educação Especial comprometida com a formação para integração em atividades, de fato, produtivas, que foquem nas habilidades do aluno.

Nota-se que o processo de compensar uma metodologia/técnica por outra é um processo de superação e de luta, o qual propicia novas vias para o desenvolvimento, ou seja, o que a criança realiza hoje com auxílio, amanhã poderá realizar sozinha.

Essa ideia está ligada ao que Vygostsky (1989) chamou de Zona de Desenvolvimento Proximal (ZDP), representando a distância entre o nível de desenvolvimento real e o nível de desenvolvimento potencial existentes na mente do sujeito, isto é, revela a diferença entre o que a criança consegue fazer sozinha e aquilo que é capaz de atingir com a ajuda de pessoas mais experientes ou em colaboração com outros sujeitos mais capacitados para tal tarefa. Podemos fazer um elo entre crianças com e sem deficiência, pois "há evidências de que a quantidade de tempo passado com os colegas sem deficiência está associada a uma variedade de benefícios acadêmicos e sociais, como uma melhor memória e melhores habilidades de linguagem e alfabetização" (Hehir et al., 2016, p. 6).

Em virtude disso, o referido teórico, argumenta que a linguagem é um fenómeno sociocultural em que o 
desenvolvimento dos processos de aquisição da linguagem é adquirido através das interações da criança com o meio ao qual encontra-se inserida.

Com efeito e como dito anteriormente, as crianças com Down, não são todas iguais, apresentam características cognitivas e psíquicas que variam de uma criança para outra, muito embora apresentem, por exemplo, características físicas muito especificas.

Desta forma, o trabalho desenvolvido com este público tem que ser individualizado e adaptado às características e ao ritmo de cada aluno. Em outras palavras, o indivíduo com Síndrome de Down, apresenta um retardo no desenvolvimento da linguagem e dificuldades de comunicação (se comparada a outra criança sem SD), características comuns a esta Síndrome.

\subsection{Abordagem cognitiva: o processo de aprendizagem}

As crianças com Síndrome de Down levam mais tempo para se desenvolver e, portanto, precisam de um currículo norteador mais específico, de propostas pedagógicas que favoreçam o aprendizado desse público. Essas crianças possuem, em geral, um perfil de aprendizagem específico caracterizado com pontos fortes e fracos. Contudo, estar por dentro dos fatores que facilitam e/ou inibem o aprendizado destes, permite aos mediadores, planejar e desenvolver atividades e sistemas alternativos de comunicação, como gestos e símbolos gráficos, proporcionando-lhes um desenvolvimento adequado, uma vez que a linguagem se origina das relações e interações sociais diárias (Silva et al., 2020).

É necessário avaliar as dificuldades de aprendizagem e as necessidades especiais inerentes ao indivíduo Down, no intuito de determinar a perspectiva intervencional a ser aplicada. Isso está ligado ao fato de que a aprendizagem depende da integração de processos neurológicos ao mesmo tempo que está relacionada à evolução de funções específicas tais como linguagem, esquema corporal, percepção e lateralidade. A par dessas limitações, é preciso estimulá-las com intervenções adequadas para que a informação seja assimilada, pois crianças com Síndrome de Down apresentam uma dificuldade maior em processar e codificar a informação.

Ainda mais, o cérebro da criança com Down precisa ser estimulado com mais informações, mesmo que o resultado se processe de uma forma mais lenta e seja visível mais tardiamente. A estimulação é um conjunto de estímulos que auxilia e se torna muito importante para o desenvolvimento cognitivo, visto que a criança inicia sua aprendizagem desde que nasce.

Assim, a intervenção precoce torna-se fundamental para o desenvolvimento da criança e para aprendizagens que vão ser construídas ao longo da vida. A criança relaciona o que aprende com os registos que tem na sua memória, ou seja, o professor ensina algo novo, a criança com Síndrome de Down aprende de maneira espontânea, relaciona o que está a aprender com os registos de memória que tem e impulsiona o exercício mental da memória.

Em uma pesquisa recente Freitas et al., (2021) aponta o interesse em realizar pesquisas que possuem o público-alvo da Educação Especial - PAEE. Nesse sentido concordamos com os autores no sentido de já existir uma literatura consolidada acerca da inclusão escolar, formação, ensino e aprendizagem, mas percebeu-se uma lacuna de estudos sobre Síndrome de Down. Nesse sentido buscamos com essa pesquisa apontar um cenário sobre a materialidade de pesquisas relacionadas a essa temática.

A motivação em pesquisar essa temática, surgiu da necessidade de ressaltar as pesquisas que foram desenvolvidas acerca da Síndrome de Down, descrevendo em quais eixos vem sendo desenvolvidas as pesquisas nessa área, e identificando os avanços e discussões que norteiam as diversas áreas do desenvolvimento. Elegemos pesquisar as pesquisas na Revista Brasileira de Educação Especial, que foi escolhida por ser um dos periódicos mais antigos sobre a área no Brasil, criada em 1993.

O interstício selecionado para o Estado da Arte refere-se aos anos de 2005 a 2020, a justificativa deve-se a ser um período em que estão disponíveis na biblioteca virtual Scielo todos os números publicados. Neste intervalo de quinze anos foram lançadas 61 edições, e estas tornaram nosso corpus de análise. 


\section{Metodologia}

Trata-se de uma revisão integrativa da literatura, a qual tem por finalidade reunir e sintetizar resultados de pesquisas acerca de um determinado tema, de maneira sistemática, contribuindo para o aprofundamento do conhecimento da área e permitindo conclusões acerca de uma questão específica (Mendes et al., 2008, Silva et al., 2021, Freitas et al., 2021).

Para conduzir o caminho percorrido na Revisão Integrativa (RI), optou-se por trabalhar com as cinco fases do processo de elaboração proposta por Cooper (1984) como pode ser observado no Quadro 1.

Quadro 1: Etapas de Elaboração da Revisão Integrativa.

\begin{tabular}{|c|l|}
\hline Etapas & \multicolumn{1}{c|}{ Processos } \\
\hline Formulação do problema & $\begin{array}{l}\text { Quais os avanços, discussões e tendências acerca das pesquisas desenvolvidas sobre } \\
\text { Síndrome de Down? }\end{array}$ \\
\hline Coleta de dados & $\begin{array}{l}\text { As buscas serão realizadas na Revista Brasileira de Educação Especial na biblioteca } \\
\text { virtual Scielo, banco de dados da revista no período de (2005-2020). }\end{array}$ \\
\hline Avaliação dos dados & $\begin{array}{l}\text { Essa etapa tem grande relevância no estudo, pois é o momento de elucidar os principais } \\
\text { achados dos pesquisadores que já investigaram a temática. A coleta das pesquisas será } \\
\text { organizada com vistas a contemplar os achados mais relevantes nos estudos analisados, } \\
\text { mantendo uma padronização que possibilitou uma análise mais fidedigna. Elegeram-se } \\
\text { alguns elementos que foram tabulados em uma planilha a partir da leitura atenta de cada } \\
\text { pesquisa, sendo eles: identificação; características metodológicas dos estudos; aspectos } \\
\text { conceituais; resultados e generalizaços; outros dados interessantes. }\end{array}$ \\
\hline $\begin{array}{c}\text { Análise e interpretação dos } \\
\text { dados }\end{array}$ & $\begin{array}{l}\text { Algumas questões centrais foram observadas, tanto no que se refere à parte teórica } \\
\text { conceitual quanto às questões metodológicas nas pesquisas publicadas, a exemplo } \\
\text { pesquisas que envolve a Família e a motricidade (desenvolvimento motor) de pessoas } \\
\text { com Síndrome de Down }\end{array}$ \\
\hline $\begin{array}{c}\text { Resultado da Análise dos } \\
\text { juízes }\end{array}$ & Verificar se os artigos respondiam à pergunta de pesquisa. \\
\hline \multicolumn{2}{|c|}{}
\end{tabular}

Fonte: Cooper (1984)

Foram analisadas 30 pesquisas, que versam sobre aspectos relacionados à Síndrome de Down, considerando as tendências e avanços das pesquisas nessa área, por meio do seguinte descritor: "Síndrome de Down”. Foram arroladas todas as pesquisas que envolviam esse descritor.

Posteriormente, foram analisadas as pesquisas que mais interessavam para compor o corpus desta revisão. Foi utilizado como critérios de inclusão, a contribuição da pesquisa, abordagem a respeito a SD e se havia uma discussão relevante sobre a deficiência. Foram excluídas, pesquisas que não se relacionavam com os objetivos desse estudo, assim como os critérios de inclusão. Dessa forma, foi identificados 30 pesquisas.

A partir dessa fase, foi possível encontrar evidências a serem utilizadas na elaboração dos resultados e discussão, para conseguinte análise e interpretação dos resultados realizada de forma descritiva.

\section{Resultados e Discussão}

No recorte realizado foram identificados trinta trabalhos envolvendo a temática em questão: pesquisas sobre Síndrome de Down, alinhada a outras áreas de conhecimento. No Quadro 2 evidenciamos o ano de publicação, autores e títulos dos estudos realizados, discriminados a seguir. 
Quadro 2: Trabalhos identificados na Revista.

\begin{tabular}{|c|c|c|}
\hline Ano & Autores & Título \\
\hline 2018 & Pelosi et. al., & $\begin{array}{l}\text { Atividades Lúdicas para o Desenvolvimento da Linguagem Oral e Escrita para Crianças e } \\
\text { Adolescentes com Síndrome de Down }\end{array}$ \\
\hline 2018 & Rocha \& Souza & $\begin{array}{l}\text { Levantamento Sistemático dos Focos de Estresse Parental em Cuidadores de Crianças com } \\
\text { Síndrome de Down }\end{array}$ \\
\hline 2018 & Leite et al., & $\begin{array}{l}\text { Controle Postural em Crianças com Síndrome de Down: Avaliação do Equilíbrio e da } \\
\text { Mobilidade Funcional }\end{array}$ \\
\hline 2018 & Spinazola et al., & $\begin{array}{l}\text { Crianças com Deficiência Física, Síndrome de Down e Autismo: Comparação de } \\
\text { Características Familiares na Perspectiva Materna na Realidade Brasileira }\end{array}$ \\
\hline 2017 & Borges et al., & Análise de Atividades Gráficas para Crianças com Síndrome de Down \\
\hline 2016 & Trindade \& Nascimento & Avaliação do Desenvolvimento Motor em Crianças com Síndrome de Down \\
\hline 2016 & Rodrigues \& Silva & $\begin{array}{l}\text { Escolarização e Institucionalização de Filhos com Síndrome de Down: Experiências de Casais } \\
\text { Idosos Portugueses }\end{array}$ \\
\hline 2016 & Barby \& Guimarães & $\begin{array}{l}\text { Desenvolvimento de Habilidades Metafonológicas e Aprendizagem da Leitura e da Escrita em } \\
\text { Alunos com Síndrome de Down }\end{array}$ \\
\hline 2015 & Rodrigues, Campos \& Almeida & Uso do PECS associado ao vídeo modeling na criança com Síndrome de Down \\
\hline 2015 & Anunciação; Costa \& Denari & $\begin{array}{l}\text { Educação Infantil e Práticas Pedagógicas para o Aluno com Síndrome de Down: o Enfoque } \\
\text { no Desenvolvimento Motor }\end{array}$ \\
\hline 2015 & Mecca et al., & Perfil de Habilidades Cognitivas Não-Verbais na Síndrome de Down \\
\hline 2015 & $\begin{array}{l}\text { Lorenzo; Braccialli \& } \\
\text { Araújo }\end{array}$ & $\begin{array}{l}\text { Realidade Virtual como Intervenção na Síndrome de Down: uma Perspectiva de Ação na } \\
\text { Interface Saúde e Educação }\end{array}$ \\
\hline 2013 & $\begin{array}{l}\text { Mayer; Almeida \& Lopes- } \\
\text { Herrera }\end{array}$ & Síndrome de Down versus alteração de linguagem: interação comunicativa entre pais e filhos \\
\hline 2012 & Sant'Anna & Conviver com a Síndrome de Down em escola inclusiva \\
\hline 2012 & Grisante \& Aiello & $\begin{array}{l}\text { Interações familiares: observação de diferentes subsistemas em família com uma criança com } \\
\text { Síndrome de Down }\end{array}$ \\
\hline 2012 & Luiz \& Nascimento & Inclusão escolar de crianças com Síndrome de Down: experiências contadas pelas famílias \\
\hline 2011 & Cavalcante & Discurso argumentativo e produção de sentidos em indivíduos com Síndrome de Down \\
\hline 2010 & Ferraz; Araújo \& Carreiro & $\begin{array}{l}\text { Inclusão de crianças com Síndrome de Down e paralisia cerebral no ensino fundamental I: } \\
\text { comparação dos relatos de mães e professores }\end{array}$ \\
\hline 2010 & Santos; Weiss \& Almeida & Avaliação e intervenção no desenvolvimento motor de uma criança com Síndrome de Down \\
\hline 2010 & Anhão; Pfeifer \& Santos & Interação social de crianças com Síndrome de Down na educação infantil \\
\hline 2009 & Macêdo et al., & $\begin{array}{l}\text { Avaliação da relação entre o déficit de atenção e o desempenho grafo-motor em estudantes } \\
\text { com Síndrome de Down }\end{array}$ \\
\hline 2009 & Macedo \& Berribille & Adultos com Síndrome de Down \\
\hline 2009 & Pereira \& Batanero & Percursos educativos, formativos e profissionais na Síndrome de Down \\
\hline 2008 & Luiz et al., & $\begin{array}{l}\text { A inclusão da criança com Síndrome de Down na rede regular de ensino: desafios e } \\
\text { possibilidades }\end{array}$ \\
\hline 2008 & Pacanaro; Santos \& Suehiro & Avaliação das habilidades cognitiva e viso-motora em pessoas com Síndrome de Down \\
\hline 2008 & Teixeira \& Kubo & $\begin{array}{l}\text { Características das interações entre alunos com Síndrome de Down e seus colegas de turma } \\
\text { no sistema regular de ensino }\end{array}$ \\
\hline 2007 & Silva \& Dessen & Crianças com e sem síndrome de Down: valores e crenças de pais e professores \\
\hline 2007 & Luiz \& Kubo & Percepções de jovens com Síndrome de Down sobre relacionar-se amorosamente \\
\hline 2006 & Silva \& Kleinhans & Processos cognitivos e plasticidade cerebral na Síndrome de Down \\
\hline 2005 & Petean \& Suguihura & Ter um irmão especial: convivendo com a Síndrome de Down \\
\hline
\end{tabular}

Fonte: Dados da Pesquisa.

Nessa pesquisa, catalogamos e identificamos que os autores são provenientes de diversas áreas não se limitando apenas a formação em educação. O título dos trabalhos já evidencia, de certa forma, o campo de atuação dos proponentes de cada pesquisa. Ao realizamos esse estudo constatamos os seguintes campos de conhecimento: Relação Familiar (6); Motricidade (6); Linguagem (4); Cognição (4); Inclusão escolar (2); Inclusão e família (2); Sociabilidade/Socialização (3); Escola (1); Formação de professores (1); Outros (1). 
Dessa forma, percebemos que pesquisas que envolve a Família e a motricidade (desenvolvimento motor) são os campos mais investigados nessa revista quando, se fala da pessoa com Síndrome de Down. Em seguida trabalhos relacionados a linguagem e a cognição. Provavelmente isso se deve, ao fato de que, a criança com Down tem um desenvolvimento motor e linguístico um pouco mais lento quando comparado àquela que não possui esta característica, nesse caso a família tem a responsabilidade de potencializar o progresso do filho(a) e buscar conhecer o perfil do familiar para que, consiga construir um vínculo afetivo, tão importante para interação/comunicação.

Figura 1: Gráfico da distribuição dos artigos publicados na RBEE no período de 2005 a 2020.

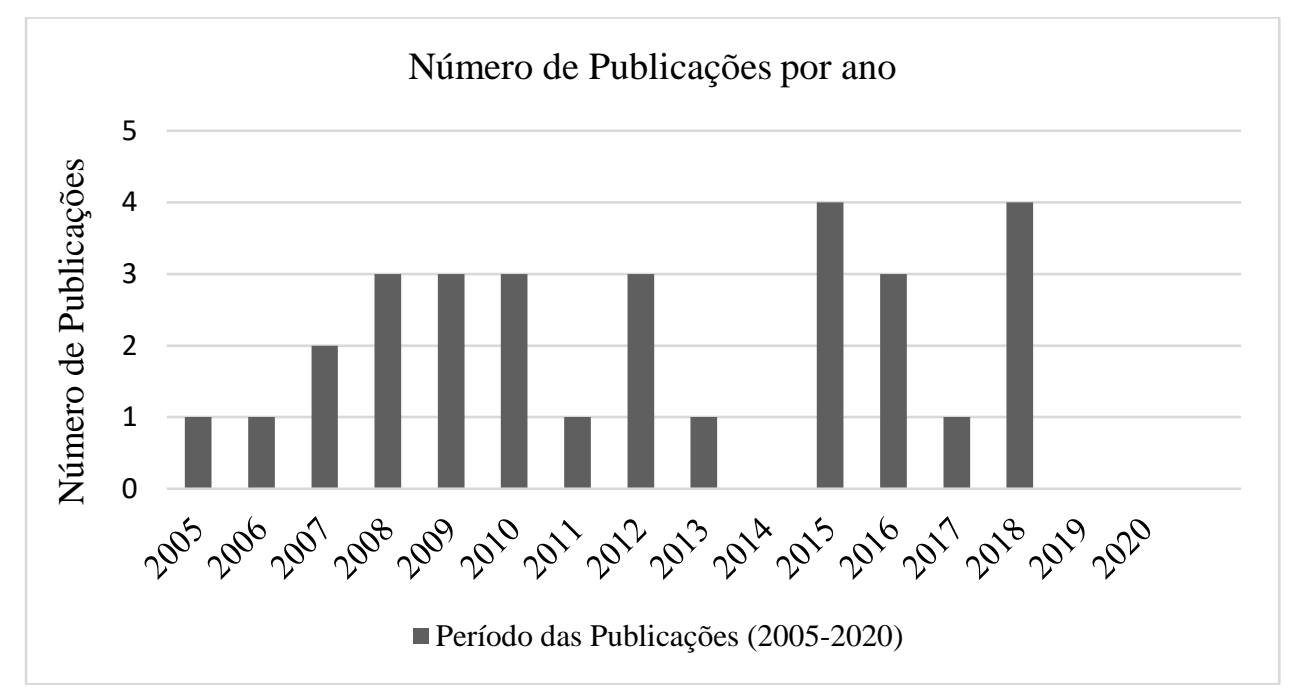

Fonte: Dados da Pesquisa.

Analisando essas produções em termos de publicação por ano observamos que houve um pequeno crescimento de pesquisas ao longo do período pesquisado (2005-2020), e que estas pesquisas em sua maioria são oriundas de universidades públicas. Uma explicação para esse resultado deve-se ao fato dessas instituições de ensino apresentarem, como aponta Silva e Amaral, (2021) condições que propiciam o desenvolvimento dessas pesquisas, como: professores pesquisadores em regime de dedicação exclusiva; boa infraestrutura; apoio financeiro das próprias universidades e de órgãos de fomento à pesquisa e grupos de pesquisa consolidados.

Outro dado que merece destaque, se refere ao número de publicações sobre a temática na revista por edição, percebemos que os anos de 2015 e 2018 tiveram expressivos estudos resultando em oito artigos, nos anos de 2014, 2019 e 2020 não houve nenhum texto que contemplasse a pessoa com síndrome de Down. Além disso é valido ressaltar que a maioria dos artigos encontrados se constituem em relatos de pesquisas, ou seja, as experiências dos autores ou pesquisas empíricas aplicando instrumentos ou recursos para descobri ou potencializar alguma habilidade do indivíduo com Down. Em menor número, identificamos trabalhos que consistiram em revisão de literatura e resenhas, conforme apresentado na tabela a seguir.

A seguir, resolvemos apresentar também a região no qual os artigos identificamos foram realizados, com intuito de retratar como esse tema tem sido pesquisado em nosso país. Identificamos que a maioria dos estudos se concentram na região sudeste com escore de 17 trabalhos, representando mais da metade das pesquisas, seguidos na região sul com sete. Notamos que estudos sobre a pessoa com Síndrome de Down nas demais regiões, não é devidamente contemplado, ao ponto que existem publicações de pesquisas feitas em outros países, como por exemplo em Portugal. 
Figura 2: Gráfico sobre a distribuição dos artigos publicados na RBEE por regiões.

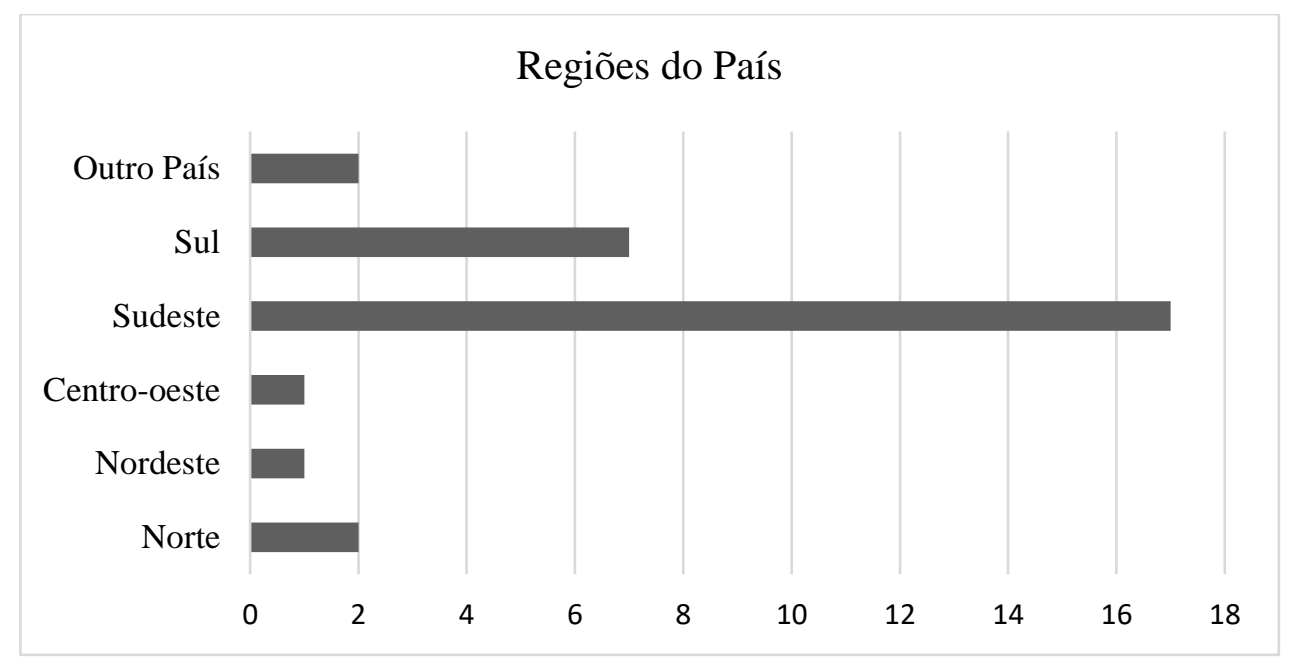

Fonte: Dados da Pesquisa.

É válido também evidenciar a abordagem metodológica identificada nas pesquisas. Em maior número, está a abordagem qualitativa sendo utilizada principalmente nas pesquisas de relato de experiência, e em seguida encontrados estudos que mesclam as duas abordagens: Quali-quantitativa, no gráfico a seguir evidenciamos os dados.

Figura 3: Gráfico sobre a abordagem metodológica das pesquisas.

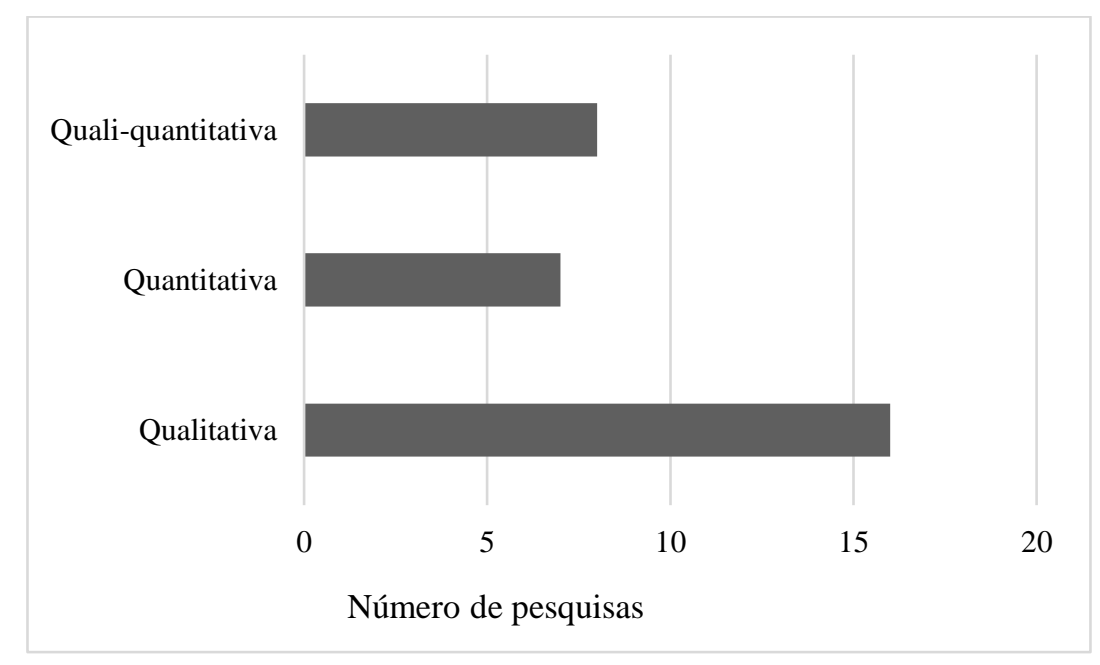

Fonte: Dados da Pesquisa.

\subsection{Focos temático das produções}

Com a finalidade de identificar os principais avanços, discussões e tendências acerca das pesquisas desenvolvidas sobre Síndrome de Down, optamos por realizar a revisão a partir da identificação dos focos temáticos como realizado por Silva \& Amaral, (2021), para a realização de nossa análise como apontado no (Quadro 3). 
Quadro 3: Focos temáticos que emergiram da investigação.

\begin{tabular}{|ll|}
\hline$*$ & Análise de desempenho, avaliação e instrumentos avaliativos para o Síndrome de Down \\
\hline$*$ & Concepções, competências, percepções e representações. \\
\hline$*$ & Metodologia/Didática de ensino para alunos com Síndrome de Down \\
\hline$*$ & Currículo na perspectiva da educação especial e inclusiva \\
\hline$*$ & Outras abordagens \\
\hline
\end{tabular}

Fonte: Silva e Amaral (2021) - Adaptado.

\subsubsection{Análise de desempenho, avaliação e instrumentos avaliativos para o Síndrome de Down}

Nos estudos de Mayer et al., (2013) houve o interesse em investigar as interações entre mães e crianças com síndrome de Down em comparação as mães que tinham filhos com atraso na linguagem. A pesquisa que se desenvolveu de forma empírica através de gravações in loco com mãe e filho. Os autores constaram que as crianças que tinham atraso da linguagem, as mães buscavam compensar essa questão, estimulando a fala, a conversar e tentando ensinar a estruturação da Língua Portuguesa, já as mães de crianças com Down focavam apenas na interação comunicativa, sem atentar as outras questões que envolvem a linguagem.

Em sequência a essa análise, os autores Trindade e Nascimento (2016) realizam uma avaliação do desenvolvimento motor, buscando identificar a idade motora de cada criança, para isso foi realizado os testes de Escala de Desenvolvimento Motor (EDM), o que foi constato que as crianças que participaram da pesquisa têm um desenvolvimento motor inferior comparado a crianças da mesma idade sem Síndrome de Down. Dessa forma, os autores reforçam a importância de estimular desde a idade mais tenra a motricidade através do apoio da família e da atuação de uma equipe multidisciplinar.

Enquanto Leite et al. (2018, p. 173), apresentam dados que objetivam "caracterizar o equilíbrio e a mobilidade funcional de crianças com SD, uma vez que possibilitam a execução de atividades do cotidiano". Os autores utilizaram dois testes para geração de dados; Escala de Equilíbrio Pediátrica (EEP) e Teste de Alcance (TA). Houve a participação de 21 crianças, nesse estudo verificou-se que o escore EEP teve um desempenho relativamente satisfatório, já em relação a TA houve um escore baixo. Através dos testes os autores consideram necessário a prática de atividades físicas e esportivas, para melhorar o equilíbrio e a mobilidade funcional.

Com o mesmo viés de análise do desenvolvimento motor, os autores Santos et al., (2010) fazem uma pesquisa descritiva através de um estudo de caso. Houve a aplicação de testes da Escala de Desenvolvimento Motor - EDM, descrevendo níveis de motricidade, equilíbrio, lateralidade dentre outros pontos. A criança foi acompanhada durante 32 sessões, ao final delas, verificou-se avanços na motricidade global, equilíbrio e organização espacial, mas sem avanços na motricidade fina, esquema corporal e a organização temporal. Assim, os autores acreditam ser relevante a participação desse público em atividades e programas de intervenção para a melhora da motricidade.

Por fim, Cavalcante (2011) analisa a produção de sentidos, através do discurso argumentativo, nesse estudo a autora realizada a pesquisa com duas crianças com Down com uso de atividades com um teor discursivo, investigando dessa forma a interpretação de texto e comunicação das participantes, na pesquisa foram usados instrumentos de comunicação alternativa, como a prancha. Através da pesquisa, constatou-se que as alunas respondiam as atividades por meio de gestos e verbalizações curtas. Assim, a pesquisadora destaca a importância de estimular a conversação e o diálogo através da comunicação alternativa. 


\subsubsection{Concepções, competências, percepções e representações}

Nos estudos de Silva e Kleinhans (2006), evidenciaram as dificuldades cognitivas e a Síndrome de Down, discutindo algumas das descobertas relacionadas aos processos cognitivos na Síndrome de Down, dando ênfase a importância da plasticidade cerebral no desenvolvimento da aprendizagem. Assim, fizeram um apanhado dos processos cognitivos na Síndrome de Down, relacionando os conceitos gerais de plasticidade cerebral e verificando como esses conhecimentos podem favorecer a aprendizagem, destacam que há diferenças entre um sujeito e outro.

A pesquisa de Luiz e Kubo (2007) apresentam as dificuldades de pais e profissionais em lidar com comportamentos de natureza sexual de pessoas com deficiência mental. O objetivo do trabalho foi descobrir quais as percepções de jovens com Síndrome de Down sobre se relacionar amorosamente. Os autores concluíram que as percepções não diferem daquelas de jovens sem síndrome e são desenvolvidas pelas oportunidades de se comportarem efetivamente sob contingências que favoreçam comportamentos amorosos. Giami (2004) mostra que os pais são radicalmente contrários à eventualidade de um bebê deficiente, e assim, frequentemente são favoráveis ao uso da esterilização como método de contracepção. Essa temática sobre relações amorosas entre pessoas com SD necessitam estar em pauta nos ambientes em que convivem para desmitificar essas concepções.

O objetivo dos estudos de Pacanaro et al., (2008) foi de avaliar habilidades intelectuais e viso-motoras em pessoas com Síndrome de Down, utilizando os testes TONI 3 (Forma A) e o Bender - Sistema de Pontuação Gradual (B-SPG). Os instrumentos demonstraram que os participantes que tiveram mais acertos na medida de inteligência foram os que cometeram menos erros na medida de habilidade viso-motora.

Os pesquisadores Macêdo et al. (2009), tiveram como objetivo avaliar a relação existente entre o déficit de atenção e o desempenho de uma conduta grafo-motora necessária à aquisição da linguagem lecto-escrita em cinco estudantes com Síndrome de Down. Como estratégia metodológica foram realizados dois testes: um de Processamento Mental para avaliar o tempo de reação a um estímulo programado e outro de avaliação das variáveis relacionadas à conduta grafo-motora. Os resultados indicaram uma estreita relação entre as duas variáveis pesquisadas, os estudantes que apresentaram um longo tempo de reação alcançaram um baixo escore no teste grafo-motor e aqueles que tiveram rápido tempo de reação obtiveram um alto escore.

Macedo e Berribille (2009), reuniram pesquisas no livro intitulado “Adultos com Síndrome de Down - A deficiência mental como produção social", (2008). O objetivo foi discutir a deficiência mental como uma condição que se desenvolve a partir das relações sociais estabelecidas com sujeitos que apresentam algum tipo de comprometimento orgânico. O livro apesar de não discutir a Síndrome de Down, traz uma ideia de que a constituição de sujeitos na condição de deficientes mentais está vinculada às relações sociais estabelecidas com eles. É uma pesquisa relevante, pois mostra experiências de vida positivas de pessoas que, mesmo apresentando dificuldades significativas, romperam com as expectativas negativas da sociedade e construíram novas possibilidades, conseguindo a independência social.

Nas análises de Anhão et al., (2010), o objetivo da pesquisa foi verificar a interação social de crianças com Síndrome de Down e crianças com desenvolvimento típico, na Educação Infantil. Os resultados apontam que, de maneira geral, não foram observadas diferenças significativamente relevantes entre os comportamentos apresentados pelo grupo de estudo e pelo grupo comparado. O estudo concluiu que nos comportamentos observados e de acordo com a faixa etária estudada, o grupo de crianças com Síndrome de Down não apresentou características de interação social muito diferentes das crianças com desenvolvimento típico. Reforçando a importância do processo de inclusão escolar desta população.

A autora Sant'Anna (2012), apresenta o resultado da pesquisa desenvolvida por Susana Couto Pimentel em seu doutorado, fazendo uso de uma análise microgenética do processo de formação de conceitos por estudantes com Síndrome de Down na escola regular. Discute a formação do pensamento conceitual a partir da atuação do professor como mediador pedagógico, demostrando como estes estudantes com síndrome de Down se apropriam, no contexto escolar, dos conceitos propostos e sistematizados pela escola no processo de educação inclusiva. 
Nas análises de Rocha e Souza (2018), buscaram compreender quem são os cuidadores principais das crianças com SD e os focos de estresse parental. Reuniram pesquisas empíricas publicadas a partir de 2010, encontradas em diferentes plataformas. Como resultado, evidenciaram que o estresse parental está relacionado à deficiência, ao desenvolvimento da criança e ao papel do cuidador. Os principais focos de estresse foram o impacto do diagnóstico; medo da discriminação e preconceito; falta de autonomia da criança; sobrecarga emocional do cuidador; preocupação com a sexualidade; baixa escolaridade paterna; mães que não podem trabalhar; e crianças mais velhas. As mães apresentaram os níveis mais elevados de estresse. Concluíram que os focos de estresse parental são diversos e causam abalo emocional, dificuldades de aceitação e adaptação familiar.

Spinazola et al. (2018), comparam as necessidades familiares e o suporte social das famílias de crianças com deficiência física, síndrome de Down e autismo, na perspectiva das mães. Como resultados, relacionado às necessidades, identificaram-se diferenças estatisticamente significativas. Concluíram que o estudo pode contribuir para conhecimento das principais necessidades e do suporte que as famílias de crianças com diferentes deficiências possuem.

Os pesquisadores Rodrigues e Silva (2016), objetivaram explorar as experiências de casais idosos portugueses com a educação e institucionalização de seus filhos com Síndrome de Down. Desenvolveram a pesquisa através do método da história oral, com a participação de catorze casais. A institucionalização foi motivo de satisfação e contentamento para alguns casais, pela oferta de oportunidades de desenvolvimento e apoio de profissionais especializados. No entanto, revestiu-se de preocupação pelo pouco e tardio investimento na aprendizagem cognitiva dos filhos, o que comprometeu o seu desenvolvimento neste aspecto para a vida social.

Os autores Silva e Dessen (2007), problematizaram os valores e as crenças de pais e professores que permeiam as práticas de cuidados e socialização das crianças e se expressam nas atividades rotineiras em que elas se engajam com seus cuidadores. Os resultados mostraram diferenças nos valores e crenças relatados pelos genitores de crianças com e sem síndrome de Down, especialmente no que se refere ao desenvolvimento motor do filho. Já as professoras relataram mudanças no desempenho acadêmico das crianças, bem como em seus relacionamentos sociais. Enquanto as professoras da Educação Especial esperam progressos nos resultados acadêmicos, em longo prazo, as professoras do ensino regular esperam resultados mais imediatos de suas crianças com desenvolvimento típico. Foi possível perceber algumas similaridades entre as crenças de pais e professoras, as quais acreditam que o apoio e o envolvimento da família podem propiciar à criança com SD os avanços necessários ao seu desenvolvimento.

O estudo de Anunciação et al., Denari (2015) buscou identificar qual o nível de desenvolvimento motor de uma criança com Síndrome de Down no contexto educacional, intervindo com estratégias de ensino necessárias para responder às necessidades de seu desenvolvimento e avaliar os avanços no seu desenvolvimento após a intervenção. Como resultado da pesquisa, perceberam que o aluno avançou em relação à faixa etária inicial referente ao período de um a dois anos para a idade entre dois a três anos em seu desenvolvimento. Nas conclusões as autoras evidenciam a necessidade da escolarização para o desenvolvimento motor e a socialização da criança com SD.

Lorenzo, Braccialli e Araújo (2015), avaliaram os resultados de intervenções com o uso da Realidade Virtual (RV) perante as necessidades psicomotoras de uma criança com Síndrome de Down. Os resultados alcançados após 20 sessões de intervenção revelaram uma melhora nas habilidades de motricidade global, equilíbrio, esquema corporal e organização espacial, entretanto o desenvolvimento da motricidade fina e linguagem/organização temporal se mantiveram estáveis. Apontaram ainda que para a possibilidade de eficácia do uso da RV, no que tange ao desenvolvimento da motricidade na SD.

Objetivou-se nos estudos de Mecca et al. (2015), verificar o desempenho de crianças com SD na Escala Internacional de Inteligência Leiter-R, comparando-as a um grupo de crianças com desenvolvimento típico, bem como os próprios desempenhos nos diferentes subtestes. Como resultados, mostraram desempenho inferior ao grupo controle em tarefas que avaliam processamento visual e raciocínio fluido. Houve diferenças de gênero somente no subteste que avalia raciocínio indutivo, 
com melhor desempenho das meninas.

\subsubsection{Metodologia/Didática de ensino para alunos com Síndrome de Down}

A Comunicação Suplementar e Alternativa (CSA) nas pesquisas de Rodrigues et al., (2015), é uma área da Tecnologia Assistiva que busca facilitar a participação das pessoas nos vários contextos comunicativos. Investigaram os efeitos do PECS associado ao VM acerca do desenvolvimento das habilidades de comunicação de uma criança com síndrome de Down com necessidades complexas de comunicação. Nos resultados indicaram que a participante aumentou as iniciações comunicativas, o vocabulário, além de demonstrar independência em utilizar o sistema PECS para se comunicar com diferentes pessoas. Os autores mostraram com os resultados uma possibilidade de intervenção que favoreceu o desenvolvimento das habilidades de comunicação da participante com síndrome de Down.

A pesquisa de Borges et al., (2017), analisou a acessibilidade das atividades gráficas aplicadas às crianças com síndrome de Down nos atendimentos terapêuticos ocupacionais, que ocorreram em uma Brinquedoteca Terapêutica, no período de um semestre. Ao final da pesquisa mostraram que os materiais gráficos estavam de acordo com as orientações da literatura no que se refere à apresentação visual, à organização do conteúdo, ao nível de leitura e ao uso de elementos para transmitir informações, mas os textos escritos com o apoio de símbolos precisavam ser aprimorados, assim como a inclusão de elementos para direcionar o local da resposta e palavras-chave em destaque no enunciado. Porém, os materiais gráficos desenvolvidos pelos terapeutas ocupacionais estavam acessíveis para a população estudada, porém não foi possível verificar outros elementos referentes à aplicação da atividade que pudessem influenciar na acessibilidade.

Barby e Guimarães (2016), investigaram os resultados produzidos pela aplicação de um programa de intervenção pedagógica que englobou o ensino do alfabeto associado ao treinamento de habilidades metafonológicas com vistas à aprendizagem da leitura e escrita. Os resultados mostram que o ensino explícito dos nomes e sons das letras, associado ao treinamento de habilidades metafonológicas e das conexões entre fonemas e grafemas possibilitou aos alunos com Síndrome de Down a compreensão do princípio alfabético e aprendizagem da leitura e da escrita. Os autores concluíram que as habilidades desenvolvidas na intervenção possibilitaram, por meio da mediação fonológica, aos participantes a aprendizagem inicial da linguagem escrita.

\subsubsection{Currículo na perspectiva da educação especial e inclusiva}

Sobre o processo de inclusão de aluno com Síndrome de Down (SD) e Paralisia Cerebral (PC), os autores Ferraz et al., (2010), fizeram uma comparação dos relatos de pais e professores e analisar como sua interação afeta o processo de inclusão. Ao final da pesquisa observaram que: oportunidade de a inclusão diminuir o preconceito; a expectativa dos pais em matricular o filho na escola regular como possibilidade de aprender a ler e a escrever ao menos o nome. Mas, os professores declaram não se sentirem preparados para este trabalho; entretanto, mesmo sem orientações, recursos e estrutura física adequada, trabalham para efetivação do aprendizado em sala de aula.

Em relação a questão de empregabilidade, os pesquisadores Pereira e Batanero (2009), em sua pesquisa objetivaram conhecer o grau de satisfação dos trabalhadores com Síndrome de Down (SD) relativamente à sua integração profissional (satisfação laboral, satisfação com o processo de integração na vida ativa). A pesquisa ocorreu em Lisboa e Vale do TejoPortugal e destacaram que para as pessoas com SD é necessário "[...]dar-lhes oportunidades reais de formação e de emprego e criar as condições legislativas e outras para que todos os que têm capacidade para tal e assim o desejem sejam inseridos no mercado de trabalho" (p. 213).

Os estudos de Luiz et. al., (2008), buscaram evidências na literatura acerca da inclusão de crianças com Síndrome de Down na rede regular de ensino. Reuniram estudos de artigos nas bases de dados PubMed e PsycINFO, utilizando as palavras- 
chaves Down syndrome, schools, mainstreaming (education), education, infant, newborn, adolescent, child e preschool, no período de 1994 a 2007. Selecionaram oito artigos e sua análise permitiu a identificação do tema: experiências e recomendações para a inclusão. Os dados desta revisão, em sua maioria provenientes de relatos de experiências, indicaram que os fatores que colaboraram ou dificultaram o processo de inclusão da criança com síndrome de Down na rede regular de ensino relacionaramse à escola, aos pais e ao professor.

\subsubsection{Outras abordagens}

Petean e Suguihura (2005), buscaram conhecer como é a relação entre irmãos mais velhos de pessoas com diagnóstico de Síndrome de Down (SD), as informações e sentimentos que possuem sobre o diagnóstico e se houve ou não modificações na dinâmica familiar e em suas próprias vidas. Concluíram após análise dos dados gráficos, que o nascimento de um irmão com SD tem consequências na rotina de vida dos irmãos mais velhos, trazendo modificações e responsabilidades para eles. Entretanto, parece ter um efeito menos dirigido para a desestrutura psicológica, sendo poucas as manifestações de sentimentos extremos, ficando apenas no nível da tristeza, quando são expressos.

Os estudos de Teixeira e Kubo (2008), abordam características da interação entre alunos com e sem necessidades especiais que possibilitaram realizar ações planejadas para a promoção de relacionamentos afetivos entre pessoas com e sem necessidades especiais e a compreensão de suas repercussões sociais. Foi constatado que quanto maior o desenvolvimento acadêmico e o grau de participação nas atividades escolares, maior será a possibilidade de o aluno com a síndrome ser considerado amigo por seus colegas. Isso significa que nas turmas investigadas há alunos que são mais reconhecidos por seus colegas de turma, tanto forma positiva quanto negativa, que os alunos com a síndrome.

Luiz e Nascimento (2012), exploraram as experiências de famílias no processo de inclusão de crianças com síndrome de Down na rede regular de ensino. Objetivaram conhecer as potencialidades e limitações vividas por essa clientela, no período de transição da instituição especializada para a escola regular e, assim, levantar as necessidades de cuidado, com vistas à promoção de saúde dessas famílias. Os resultados evidenciaram a necessidade de acompanhamento das famílias, antes, durante e após a inclusão propriamente dita, de modo a apoiá-las nos momentos de busca e escolha da escola, de adaptação da criança ao novo ambiente e de transição dos atendimentos oferecidos pela instituição especializada para outros setores.

Os pesquisadores Grisante e Aiello (2012), pesquisaram sobre as interações familiares de crianças com Síndrome de Down (SD) que são escassos e enfatizam, quase exclusivamente, interações da mãe com a criança, apesar de a literatura ressaltar a importância de se incluir vários subsistemas familiares para um entendimento mais amplo da dinâmica familiar. Nessa pesquisa investigaram os padrões da interação entre uma criança com SD e seus familiares (pai, mãe, avó materna, irmã) em função dos subsistemas diádicos, triádicos e poliádicos; e caracterizaram alguns fatores que possivelmente têm influência sobre os padrões de interação: nível socioeconômico, níveis de estresse, rede de apoio e ajustamento marital. Os resultados apontam que membros da família são as principais fontes de apoio social, os familiares possuem baixos índices de stress e um bom ajustamento marital.

Como descrito por Silva et al., (2020) trabalhar na perspectiva da educação inclusiva com esses estudantes salienta mudanças importante, pois, se outrora, eram atendidos exclusivamente por escolas especiais, agora pela abordagem da inclusão, a meta é que esses alunos frequentem as escolas regulares, tendo o apoio da equipe do Atendimento Educacional Especializado em Sala de Recursos Multifuncionais.

\section{Considerações Finais}

Conforme discutido, a pessoa com Síndrome de Down apresenta uma alteração no número de autossomos, tendo como consequência um atraso no desenvolvimento cognitivo, linguístico e motor da criança, necessitando assim de intervenções específicas para que as habilidades do sujeito sejam desenvolvidas de forma significativa. 
Nessa perspectiva, objetivou-se com este estudo revisar a literatura que discutem a despeito das dificuldades que passam ou que possam interferir na aquisição e desenvolvimento da linguagem da pessoa com Síndrome de Down, afinal, é pela linguagem que nos constituímos enquanto seres humanos críticos-reflexivos no contexto ao qual nos inserimos, isto é, ao passo que criamos a linguagem somos também produtos dela.

Com base na revisão integrativa de literatura, pôde-se constatar que a criança com SD apresentam consideráveis dificuldades no desenvolvimento da linguagem, bem como da família e dos professores em lidar com esse sujeito, assim, é indispensável que a família, escola e outros profissionais que acompanham o sujeito com Down construam uma relação baseada no trabalho colaborativo, de modo que os diversos saberes sejam considerados possibilitando situações de aprendizagem significativas no que cerne a aquisição da linguagem.

Desse modo, são necessárias intervenções específicas que devem ser planejadas, desenvolvidas e avaliadas colaborativamente, tendo como início uma avaliação diagnóstica que possibilite compreender o nível de desenvolvimento da linguagem em que a criança com Síndrome de Down se encontra.

Assim, destacamos a seguir algumas estratégias a serem utilizadas: que a família construa diálogos conforme o nível de compreensão linguística da criança com SD e com o passar do tempo aumente o nível de complexidade; ler diariamente para a criança aumente seu vocabulário, compreenda as organizações sintáticas da língua; que os professores trabalhem com uma diversidade de gêneros textuais e portadores de texto, podendo fazer uso de tecnologias Assistivas conforme a necessidade do aluno; possibilitar à criança com SD o contato com outras crianças com e sem a síndrome, para que tenham contato com diferentes linguagens por meio de brincadeiras, jogos, danças e músicas; caso necessário, fazer uso de comunicação alternativa.

\section{Referências}

Anhão, P. P. G., Pfeifer, L. I., \& Santos, J. L. D. (2010). Interação social de crianças com Síndrome de Down na educação infantil. Revista Brasileira de Educação Especial, 16, 31-46.

Anunciação, L. M. R. L., Costa, M. P. R. D., \& Denari, F. E. (2015). Educação Infantil e Práticas Pedagógicas para o Aluno com Síndrome de Down: o Enfoque no Desenvolvimento Motor. Revista Brasileira de Educação Especial, 21, 229-244.

Barby, A. A. D. O. M., \& Guimarães, S. R. K. (2016). Desenvolvimento de habilidades metafonológicas e aprendizagem da leitura e da escrita em alunos com Síndrome de Down. Revista Brasileira de Educação Especial, 22, 381-398.

Borges, A. L. E., Pelosi, M. B., Nascimento, J. S., \& Melo, J. V. D. (2017). Análise de atividades gráficas para crianças com Síndrome de Down. Revista Brasileira de Educação Especial, 23, 577-594.

Cavalcante, T. C. F. (2011). Discurso argumentativo e produção de sentidos em indivíduos com síndrome de Down. Revista Brasileira de Educação Especial, 17, 427-440.

Cooper, H. M. (1988). The integrative research review: a systematic aproach. The integrative research review: A systematic aproach, $144-144$.

Ferraz, C. R. A., Araújo, M. V. D., \& Carreiro, L. R. R. (2010). Inclusão de crianças com Síndrome de Down e paralisia cerebral no ensino fundamental I: comparação dos relatos de mães e professores. Revista Brasileira de Educação Especial, 16, 397-414.

Freitas, T. N, de Araújo, N. F. M., da Silva Sousa, D. L., Medeiros, A. M., de Araújo Júnior, M. A., da Silva, I. R., \& Silva, R. S. (2021). Avaliação Escolar na Educação Especial: Um mapeamento na Revista Brasileira de Educação Especial no período de (2006-2020). Research, Society and Development, 10 (6), e59310616334-e59310616334.

Galvão Filho, T. A. (2012). Tecnologia Assistiva: favorecendo o desenvolvimento e a aprendizagem em contextos educacionais inclusivos. In : Giroto, C. R. M.; Poker, R. B.; Omote, S. (Org.) As tecnologias nas práticas pedagógicas inclusivas. Cultura Acadêmica, 65-92.

Grandin, A. B. (2010). Aspectos do desenvolvimento da linguagem de um grupo de crianças com síndrome de Down em contexto terapêutico grupal. (Dissertação de Mestrado). Campinas: Universidade Estadual de Campinas

Grisante, P. C., \& Aiello, A. L. R. (2012). Interações familiares: observação de diferentes subsistemas em família com uma criança com Síndrome de Down. Revista Brasileira de Educação Especial, 18(2), 195-212.

Hehir, T., Grindal, T., Freeman, B., Lamoreau, R., Borqvaye, Y., \& Burke, S. (2016). Os benefícios da educação inclusiva para estudantes com e sem deficiência. São Paulo: Instituto LANA.

Leite, J. C., Neves, J. C. D. J., Vitor, L. G. V., \& Fujisawa, D. S. (2018). Controle Postural em Crianças com Síndrome de Down: Avaliação do Equilíbrio e da Mobilidade Funcional. Revista Brasileira de Educação Especial, 24, 173-182. 
Lorenzo, S. M. D., Braccialli, L. M. P., \& Araújo, R. D. C. T. (2015). Realidade Virtual como Intervenção na Síndrome de Down: uma Perspectiva de Ação na Interface Saúde e Educação1. Revista Brasileira de Educação Especial, 21, 259-274.

Luiz, E. C., \& Kubo, O. M. (2007). Percepções de jovens com Síndrome de Down sobre relacionar-se amorosamente. Revista brasileira de Educação Especial, 13, 219-238.

Luiz, F. M. R., \& Nascimento, L. C. (2012). Inclusão escolar de crianças com síndrome de down: experiências contadas pelas famílias. Revista brasileira de educação especial, 18, 127-140.

Luiz, F. M. R., Bortoli, P. S. D., Floria-Santos, M., \& Nascimento, L. C. (2008). A inclusão da criança com Síndrome de Down na rede regular de ensino: desafios e possibilidades. Revista Brasileira de Educação Especial, 14, 497-508.

Macêdo, L., Lima, I., Cardoso, F., \& Beresford, H. (2009). Avaliação da relação entre o déficit de atenção e o desempenho grafo-motor em estudantes com Síndrome de Down. Revista Brasileira de Educação Especial, 15(3), 431-440.

Macedo, N. N., \& Berribille, G. R. (2009). Adultos com Síndrome de Down. Revista Brasileira de Educação Especial; 15(3); 501-502.

Martinho, L. S. T. (2011). Comunicação e linguagem na síndrome de down (Master's thesis).

Mayer, M. G. G., Almeida, M. A., \& Lopes-Herrera, S. A. (2013). Síndrome de Down versus alteração de linguagem: interação comunicativa entre pais e filhos. Revista Brasileira de Educação Especial, 19, 343-362.

Mecca, T. P., Morão, C. P. D. A. B., Silva, P. B. D., \& Macedo, E. C. D. (2015). Perfil de Habilidades Cognitivas Não-Verbais na Síndrome de Down. Revista Brasileira de Educação Especial, 21, 213-228.

Mendes, K. D. S., Silveira, R. C. D. C. P., \& Galvão, C. M. (2008). Revisão integrativa: método de pesquisa para a incorporação de evidências na saúde e na enfermagem. Texto \& contexto-enfermagem, 17, 758-764.

Pacanaro, S. V., Santos, A. A. A. D. \& Suehiro, A. C. B. (2008). Avaliação das habilidades cognitiva e viso-motora em pessoas com Síndrome de Down. Revista Brasileira de Educação Especial, 14, 311-326.

Pelosi, M. B., Silva, R. M. P. D., Santos, G. D., \& Reis, N. H. (2018). Atividades lúdicas para o desenvolvimento da linguagem oral e escrita para crianças e adolescentes com Síndrome de Down. Revista Brasileira de Educação Especial, 24, 535-550.

Pereira, M. E. S., \& Fernández Batanero, J. M. (2009). Percursos educativos, formativos e profissionais na Síndrome de Down. Revista Brasileira de Educação Especial, 15(2), 197-218.

Pereira-Silva, N. L., \& Dessen, M. A. (2007). Crianças com e sem síndrome de Down: valores e crenças de pais e professores. Revista Brasileira de Educação Especial, 13, 429-446.

Petean, E. B. L., \& Suguihura, A. L. M. (2005). Ter um irmão especial: convivendo com a Síndrome de Down. Revista Brasileira de Educação Especial, 11, 445-460.

Piaget, J. (1999). A linguagem e o pensamento da criança. Martins Fontes.

Rocha, D. S. D. P., \& Souza, P. B. M. D. (2018). Levantamento Sistemático dos Focos de Estresse Parental em Cuidadores de Crianças com Síndrome de Down. Revista Brasileira de Educação Especial, 24, 455-464.

Rodrigues, M. N. M., \& Silva, A. L. D. (2016). Escolarização E institucionalização de Filhos com síndrome de down: Experiências de casais idosos portugueses. Revista Brasileira de Educação Especial, 22, 477-492.

Rodrigues, V., Campos, J. A. D. P. P., \& Almeida, M. A. (2015). Uso do Pecs associado ao Vídeo Modeling Na Criança Com Síndrome De Down. Revista Brasileira de Educação Especial, 21, 379-392.

Sant'Anna, M. M. M. (2012). Conviver com a Síndrome de Down em escola inclusiva. Revista Brasileira de Educação Especial; 18(4); 709-711.

Santos, A. P. M. D., Weiss, S. L. I., \& Almeida, G. M. F. D. (2010). Avaliação e intervenção no desenvolvimento motor de uma criança com síndrome de Down. Revista Brasileira de Educação Especial, 16, 19-30.

Schwartzman, José Salomão. (2003). Síndrome de Down. (2a ed.), Memnon: Mackenzie.

Silva, I. G. (2015). Vigotski, defectologia e processo educativo. Revista Pleiade, 9 (17), 77-82.

Silva, I. R., de Miranda, M. C. B. C., Branco, Y. R. B. C., \& Silva, R. S. (2021). Caracterização Bioquímica das Doenças Mitocondriais: uma revisão integrativa em teses e dissertações no período de (2000 a 2020). Research, Society and Development, 10 (11), e151101119320-e151101119320.

Silva, I. R., Freitas, T. N., de Araújo, N. F. M., da Silva Sousa, D. L., de Araújo Júnior, M. A., Medeiros, A. M., \& Silva, R. S. (2021). Acessibilidade digital em tempos de ensino remoto. Research, Society and Development, 10 (4), e60010414966-e60010414966.

Silva, M. D. F. M. C., \& Kleinhans, A. C. D. S. (2006). Processos cognitivos e plasticidade cerebral na Síndrome de Down. Revista Brasileira de Educação Especial, 12, 123-138.

Silva, R. S., \& Amaral, C. L. C. (2020). A Educação Inclusiva no Ensino De Ciências e Matemática: Um Mapeamento Na Revista Educação Especial no Período De 2000 A 2018. Communitas, 4(7), 281-294.

Silva, R. S., \& Costa Amaral, C. L. (2021). As contribuições da defectologia e da Teoria Histórico-Cultural no Ensino de Química para o deficiente visual: um estado da arte em teses e dissertações (2000-2019). Communitas, 5(9), 346-364. 
Research, Society and Development, v. 10, n. 17, e125101724409, 2021

(CC BY 4.0) | ISSN 2525-3409 | DOI: http://dx.doi.org/10.33448/rsd-v10i17.24409

Silva, R. S., de Sousa, M. V., \& da Silva, I. R. (2020). Inclusão escolar de crianças com Síndrome de Down na educação infantil. Revista Amor Mundi, 1(3), 3546.

Spinazola, C. D. C., Cia, F., Azevedo, T. L. D., \& Gualda, D. S. (2018). Crianças com Deficiência Física, Síndrome de Down e Autismo: Comparação de Características Familiares na Perspectiva Materna na Realidade Brasileira. Revista Brasileira de Educação Especial, 24, 199-216.

Teixeira, F. C., \& Kubo, O. M. (2008). Características das interações entre alunos com Síndrome de Down e seus colegas de turma no sistema regular de ensino. Revista Brasileira de Educação Especial, 14, 75-92.

Trindade, A. S., \& Nascimento, M. A. D. (2016). Avaliação do desenvolvimento motor em crianças com síndrome de down. Revista Brasileira de Educação Especial, 22, 577-588.

Vygotsky L.S. (1997). Fundamentos em Defectologia. Havana, Ed. Pueblo y Educación,

Vygotsky, L. S. (1989). A formação social da mente. (3a ed.), Martins Fontes, 1989. 